\title{
Gorlin Goltz Syndrome: Beware of Melanoma
}

\author{
Filomena Russo ${ }^{1}$, Flavio Giulio Liso ${ }^{1}$, Francesco Santi ${ }^{1}$, Luca Provvidenziale ${ }^{1}$, \\ Paolo Taddeucci ${ }^{1}$, Pietro Rubegni ${ }^{1}$
}

1 Department of Medical, Surgical and Neurological Science, Dermatology Section, University of Siena, S. Maria alle Scotte Hospital, Siena, Italy

Key words: Gorlin Goltz syndrome, melanoma, reflectance confocal microscopy, dermoscopy

Citation: Russo F, Liso FG, Santi F, Provvidenziale L, Taddeucci P, Rubegni P. Gorlin goltz syndrome: beware of melanoma. Dermatol Pract Concept. 2022;12(1):e2022038. DOI: https://doi.org/10.5826/dpc.1201a38

Accepted: July 5, 2021; Published: January 2022

Copyright: $(2022$ Russo et al. This is an open-access article distributed under the terms of the Creative Commons Attribution License BY-NC-4.0, https://creativecommons.org/licenses/by-nc/4.0/, which permits unrestricted noncommercial use, distribution, and reproduction in any medium, provided the original authors and source are credited.

Funding: None.

Competing interests: None.

Authorship: All authors have contributed significantly to this publication

Corresponding author: Francesco Santi, MD, Dermatology Section, University of Siena, S. Maria alle Scotte Hospital, Siena, Italy. E-mail: santifrancesco90@gmail.com

\section{Introduction}

Gorlin Goltz syndrome, also known as basal cell nevus syndrome, is a rare autosomal dominant multisystem disorder. The "signature" feature of Gorlin Goltz syndrome is an earlier onset and a higher number of Basal Cell Carcinomas (BCC), median of 160 BCCs in an average lifetime [1]. The genetic base of this syndrome is mainly to be found in pathologic constitutive activation of the Sonic Hedgehog signaling pathway. Patients with this syndrome, beyond numerous BCCs, can also present a wide spectrum of manifestations such as: jaw odontogenic keratocysts, palmoplantar pits, lamellar calcification of the falx cerebri, skeletal abnormalities, childhood medulloblastomas and cardiac or ovarian fibromas.

\section{Case Presentation}

A 60-year-old Caucasian woman was referred to our department because she had been diagnosed with Gorlin Goltz syndrome, with a 20-year history of multiple BCCs predominantly involving her face and upper back as well as multiple jaw cysts. After she had undergone multiple surgical procedures, she started vismodegib with a daily dose of $150 \mathrm{mg}$ from November 2019. Clinical follow-up after 3 months showed progressive improvement in the size of the lesions. On a further follow-up visit in March 2020, the patient continued to show improvement, but a 7-mm pigmented oval-shaped lesion was noted on her scalp (Figure 1A). On 20x polarized dermoscopy regression structures, irregular dark blue pigmented blotches and a brownish peripheral network reminiscent of leaf-like structures were seen, leading to a possible diagnosis of BCC versus melanoma (Figure 1B). However, reflectance confocal microscopy (RCM, Vivascope 3000, Caliber) examination showed pagetoid infiltration of round and dendritic atypical cells in the epidermis and irregular meshwork at the dermo-epidermal junction (Figure 1C), suggesting the diagnosis of melanoma. An excisional biopsy was performed, and the histopathological examination revealed an invasive melanoma with 0.48 Breslow thickness with conspicuous regression (>75\%). 


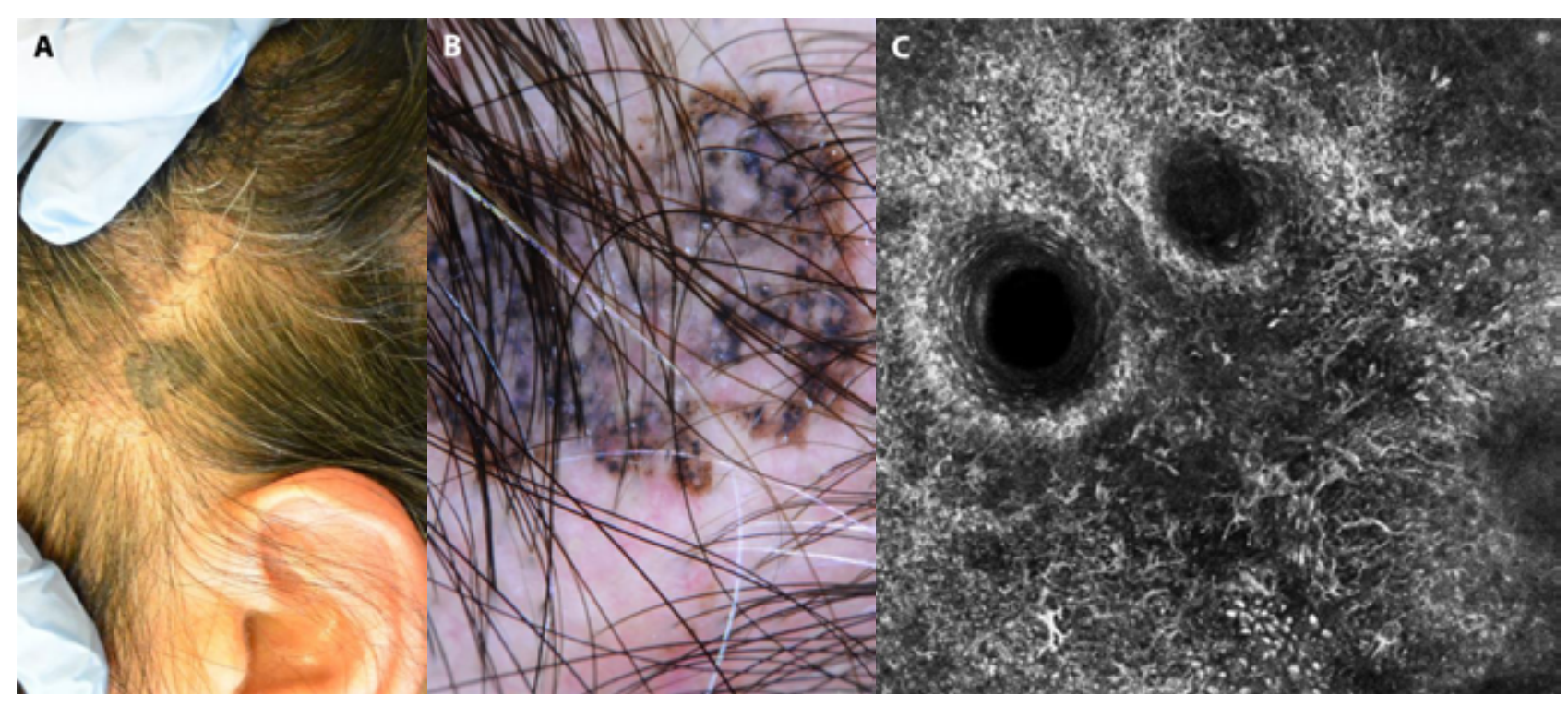

Figure 1. (A) Clinical and (B) dermoscopy aspect. 20x polarized dermoscopy shows regression structures, irregular dark blue pigmented blotches and a brownish peripheral network reminiscent of leaf-like structures. (C) Reflectance confocal microscopy (RCM) examination showed pagetoid infiltration of round and dendritic atypical cells in the epidermis.

\section{Conclusion}

The presence of melanomas in Gorlin Goltz syndrome is rare and there are only a few documented cases in the literature [2]. Since melanomas of the scalp frequently display nonclassic melanoma dermoscopic criteria they can simulate other tumors, amongst which BCC is one. Indeed, typical dermoscopic features of pigmented basal cell carcinomas such as large blue-gray ovoid nests and arborizing vessels can also be found in melanoma. RCM is able to diagnose BCCs mimicking melanoma at dermoscopy as well as melanoma mimicking BCCs. Typically, BCCs on RCM display the presence of tight basaloid islands, peripheral clefting and increased dermal vasculature whereas melanoma of the scalp usually, under RCM, has irregular meshwork patterns associated with the presence of nests of atypical melanocytes at the dermo-epidermal junction. The systematic use of RCM on these multiple inconspicuous lesions has enabled clinicians to arrive at a diagnosis of BCC or a melanocytic lesion in few minutes, and with a high level of confidence, since clear-cut confocal criteria can be observed. Our case highlights the benefits of using a combined approach between dermoscopy and
RCM in Gorlin Goltz syndrome patients to identify ambiguous lesions in order to discriminate melanoma from BCC, and therefore avoid therapeutical errors and allow clinicians to act promptly. Nonetheless this case highlights the importance of performing a complete body examination. Lesions in areas difficult to explore such as the scalp, in particular in non-bald patient where the presence of hair may hinder a quick examination, could be easily failed to spot resulting in a delayed treatment.

\section{References}

1. Solis DC, Kwon GP, Ransohoff KJ, et al. Risk Factors for Basal Cell Carcinoma Among Patients With Basal Cell Nevus Syndrome: Development of a Basal Cell Nevus Syndrome Patient Registry. JAMA Dermatol. 2017;153(2):189-192. DOI: 10.1001/jamadermatol.2016.4347. PMID: 27902821..

2. Briatico G, Moscarella E, Ronchi A, Procaccini EM, Argenziano G. In Situ Melanoma Collision With a Basal Cell Carcinoma in a Patient With Basal Cell Nevus Syndrome: Clinical and Dermoscopic Features. Dermatol Pract Concept. 2019;9(4): 310-312. DOI: 10.5826/dpc.0904a16. PMID: 31723471. PMCID: PMC6830545.. 